\title{
REGULARITY RESULTS FOR LOCAL MINIMIZERS OF ENERGIES WITH GENERAL DENSITIES HAVING SUPERQUADRATIC GROWTH
}

\author{
M. FUCHS
}

\begin{abstract}
Variational integrals whose energy densities are represented by $N$ functions $h$ of at least quadratic growth are considered. Under rather general conditions on $h$, almost everywhere regularity of vector-valued local minimizers is established, and it is possible to include the case of higher order variational problems without essential changes in the arguments.
\end{abstract}

\section{$\S 1$. INTRODUCTION}

In recent years, increasing attention has been paid to the study of the regularity properties of local minimizers $u: \mathbb{R}^{n} \supset \Omega \rightarrow \mathbb{R}^{M}$ for variational integrals of the form

$$
I[w, \Omega]=\int_{\Omega} H(\nabla w) d x
$$

where the density $H: \mathbb{R}^{n M} \rightarrow[0, \infty)$ is a strictly convex function of "nonstandard growth", which means, loosely speaking, that we do not have an ellipticity estimate of the form $\lambda(|Z|)|Y|^{2} \leq D^{2} H(Z)(Y, Y) \leq \Lambda(|Z|)|Y|^{2}$ for all $Y$ and $Z \in \mathbb{R}^{n M}$, with functions $\lambda, \Lambda$ satisfying

$$
c_{1} \leq \Lambda(t) / \lambda(t) \leq c_{2}
$$

for all $t \geq 0$ and with constants $c_{1}, c_{2}>0$. One major class showing such a behavior is generated by the so-called integrands of anisotropic power growth, e.g., $H(\nabla u)=$ $\left(1+|\nabla u|^{2}\right)^{p / 2}+\left(1+\left|\partial_{n} u\right|^{2}\right)^{q / 2}$ with exponents $p<q$, for which $\lambda(t)=a\left(1+t^{2}\right)^{\frac{p-2}{2}}$ and $\Lambda(t)=A\left(1+t^{2}\right)^{\frac{q-2}{2}}$ are functions characterizing the growth of $D^{2} H$.

In this setting for the scalar case (i.e., $M=1$ ), in [Ma1 and Ma4, Marcellini proved the interior $C^{1, \alpha}$-regularity of the local minimizers under conditions of the form $q<c(n) p$ relating the exponents $p$ and $q$, where $c(n)$ is rather large for low dimensions $n$, but $c(n) \searrow 1$ as $n \rightarrow \infty$. As a matter of fact - without further hypotheses on $H$ - in the general vector case $(M \geq 2)$ one can only hope for almost everywhere regularity. Here we mention the important contributions of Passarelli Di Napoli and Siepe [PS], of Acerbi and Fusco $\mathrm{AF}$ ] and of Esposito, Leonetti and Mingione [ELM1, ELM2 as well as the references mentioned in those papers. In addition, also the publications [BF1, BF2] are devoted to partial regularity theory for variational problems with anisotropic $(p, q)$ growth, and it should be emphasized that in all the above-mentioned papers an inequality such as $q<c(n) p$ is needed again.

A second class of integrands with nonstandard behavior arises if $H$ satisfies

$$
H(Z)=h(|Z|), \quad Z \in \mathbb{R}^{n M},
$$

2010 Mathematics Subject Classification. Primary 49N60.

Key words and phrases. Vector-valued problems, local minimizers, nonstandard growth, partial regularity. 
for a given $N$-function $h$. In this case we have

$$
\lambda(t)=\min \left\{\frac{h^{\prime}(t)}{t}, h^{\prime \prime}(t)\right\}, \quad \Lambda=\max \{\ldots\}
$$

(compare v) below), so that, in general, the uniform ellipticity condition is violated. Concentrating on the vector case, the interior $C^{1, \alpha}$-regularity of local minimizers of integrals depending on the modulus of the gradient was proved by many authors, starting with the paper of Uhlenbeck [Uh] on the $p$-growth case, i.e., $h(t)=t^{p}$ for some $p>1$, which was extended later by Giaquinta and Modica GM. Marcellini and Papi studied very general $N$-functions $h$ in Ma1, Ma2, Ma3 and MP, whereas the case of nearly linear growth, i.e., $h(t)=t \ln (1+t)$, is due to Mingione and Siepe MS. Further contributions concerning regularity problems under condition (1.2) were given in the recent paper $\mathrm{ABF}$.

The purpose of the present paper is threefold.

1. In Theorem 1.1 below we establish almost everywhere regularity for vector-valued local minimizers, requiring (1.2), but under (in a sense to be made precise) more general hypotheses such as those used in $\mathrm{MP}$.

2. Our methods apply to variational problems of higher order (see Theorem 1.2), providing some extensions of the results obtained in $\mathrm{ApF}$.

3. We think that, with some additional work, our results can be transferred to local minima $u: \mathbb{R}^{n} \supset \Omega \rightarrow \mathbb{R}^{n}$ of integrals such as $\int_{\Omega} h(|\varepsilon(u)|) d x$ subject to the constraint $\operatorname{div} u=0$. Here $\varepsilon(u)$ is the symmetric gradient of $u$, and for $n=2$ this version of the stationary Stokes problem was the subject of the recent paper [Fu2]. To our knowledge, the hypothesis (1.2) seems to be a natural assumption for the study of fluids: it occurs for example in the setting of electrorheological fluids, for which partial regularity was proved by Acerbi and Mingione in [AM].

With respect to 1, our result is not optimal: we expect that the singular set is empty, but we could not rule out the occurrence of singular points. For variational problems of higher order or in the framework of fluids, it seems to be even harder to develop methods that use the structure condition (1.2) in order to exclude singularities.

Here is a precise formulation of our assumptions. Let $n \geq 3$, let $M \geq 1$, and consider an open set $\Omega \subset \mathbb{R}^{n}$. Let the density $H: \mathbb{R}^{n M} \rightarrow[0, \infty)$ satisfy (1.2), where $h:[0, \infty) \rightarrow$ $[0, \infty)$ is of class $C^{2}$. We shall impose the following hypotheses on $h$ :

$h$ is strictly monotone increasing and convex, together with

$$
h^{\prime \prime}(0)>0 \quad \text { and } \quad \lim _{t \downarrow 0} \frac{h(t)}{t}=0 \text {; }
$$

there exists a constant $\bar{k}>0$ such that $h(2 t) \leq \bar{k} h(t)$ for all $t \geq 0$;

$$
\left\{\begin{array}{l}
\text { for an exponent } \omega \geq 0 \text { and a constant } a \geq 0 \text { we have } \\
\frac{h^{\prime}(t)}{t} \leq h^{\prime \prime}(t) \leq a\left(1+t^{2}\right)^{\frac{\omega}{2}} \frac{h^{\prime}(t)}{t} \text { for all } t \geq 0 .
\end{array}\right.
$$

Some comments on (A1)-(A3) are in order.

i) We have $h(0)=h^{\prime}(0)$, and, by convexity, $h^{\prime}$ is an increasing function with $h^{\prime}(t)>0$ for all $t>0$ : otherwise it would follow that $h^{\prime}=0$ on some interval $\left[0, t_{0}\right], t_{0}>0$, contradicting the first part of (A1).

ii) The inequality $\frac{h^{\prime}(t)}{t} \leq h^{\prime \prime}(t)$ implies that the function $t \mapsto \frac{h^{\prime}(t)}{t}$ is monotone increasing; moreover, we deduce the lower bound

$$
h(t) \geq \frac{1}{2} h^{\prime \prime}(0) t^{2}, \quad t \geq 0 .
$$


(A1) combined with (1.3) shows that $h$ is an $N$-function in the sense of [Ad, Subsection 8.2].

iii) (A2) states that $h$ satisfies a global $(\Delta 2)$-condition, and it is easily seen that

$$
h(t) \leq c\left(t^{m}+1\right)
$$

for a suitable exponent $m \geq 2$ and a constant $c$. Then, the convexity of $h$ implies that $h^{\prime}(t)$ can be bounded in terms of $t^{m-1}$.

iv) (A2) and the convexity of $h$ allow us to deduce the inequality

$$
\bar{k}^{-1} h^{\prime}(t) t \leq h(t) \leq t h^{\prime}(t), \quad t \geq 0 .
$$

v) From (1.2) it follows that, for all $Y, Z \in \mathbb{R}^{n M}$,

$\min \left\{\frac{h^{\prime}(|Z|)}{|Z|}, h^{\prime \prime}(|Z|)\right\}|Y|^{2} \leq D^{2} H(Z)(Y, Y) \leq \max \left\{\frac{h^{\prime}(|Z|)}{|Z|}, h^{\prime \prime}(|Z|)\right\}|Y|^{2}$.

Hence, by (A3),

$$
\frac{h^{\prime}(|Z|)}{|Z|}|Y|^{2} \leq D^{2} H(Z)(Y, Y) \leq a\left(1+|Z|^{2}\right)^{\frac{\omega}{2}} \frac{h^{\prime}(|Z|)}{|Z|}|Y|^{2} .
$$

Recalling iii) and using $\frac{h^{\prime}(|Z|)}{|Z|} \geq h^{\prime \prime}(0)$ (see ii)), we see that (1.5) with exponent $q:=m+\omega$ implies that

$$
h^{\prime \prime}(0)|Y|^{2} \leq D^{2} H(Z)(Y, Y) \leq C\left(1+|Z|^{2}\right)^{\frac{q-2}{2}}|Y|^{2},
$$

and (1.6) means that $H$ is of anisotropic $(2, q)$-growth.

Definition 1.1. A function $u \in W_{1, \text { loc }}^{1}\left(\Omega ; \mathbb{R}^{M}\right)$ is a local minimizer of the energy $I$ (see (1.1) ) if for any subdomain $\Omega^{\prime}$ with compact closure in $\Omega$ we have $I\left[u, \Omega^{\prime}\right]<\infty$ and $I\left[u, \Omega^{\prime}\right] \leq I\left[v, \Omega^{\prime}\right]$, where $v$ is an arbitrary function in $W_{1, \mathrm{loc}}^{1}\left(\Omega ; \mathbb{R}^{M}\right)$ such that $\operatorname{spt}(u-v) \subset \Omega^{\prime}$.

For a definition of the Sobolev spaces $W_{p, \text { loc }}^{k}\left(\Omega ; \mathbb{R}^{M}\right)$ and related classes, we refer the reader to the monograph $\mathrm{Ad}$. Note that a local minimizer actually belongs to the Orlicz-Sobolev class $W_{h, \text { loc }}^{1}\left(\Omega ; \mathbb{R}^{M}\right)$; in particular, the local minimizers are in the space $W_{2, \text { loc }}^{1}\left(\Omega ; \mathbb{R}^{M}\right)($ see $(1.3))$.

Now we state our first result.

Theorem 1.1. Assuming (A1)-(A3), consider a local minimizer $u \in W_{1, \text { loc }}^{1}\left(\Omega ; \mathbb{R}^{M}\right)$ of the functional $I$ as in (1.1) with integrand $H$ defined in (1.2). Next, suppose that there is a finite constant $c$ such that

$$
t^{\omega} \leq c\left[h(t)^{\frac{2}{n-2}}+1\right]
$$

for all sufficiently large $t$. Then there is an open subset $\Omega_{0}$ of $\Omega$ with full Lebesgue measure such that $u \in C^{1, \alpha}\left(\Omega_{0} ; \mathbb{R}^{M}\right)$ for any $0<\alpha<1$.

Remark 1.1. In accordance with (1.3), relation (1.7) is fulfilled if we require $\omega \leq 4 /(n-2)$.

Remark 1.2. Let us compare Theorem 1.1 with the recent result obtained by Marcellini and Papi $\mathrm{MP}$. Roughly speaking, they replace the second inequality in (A3) by the requirement

$$
h^{\prime \prime}(t) \leq \mathrm{const}\left(\frac{h^{\prime}(t)}{t}\right)^{\frac{n}{n-1}}
$$


(compare (2.9) of [MP]). Then, full interior regularity is established, i.e., $\Omega_{0}=\Omega$, whereas we could not exclude the occurrence of singular points despite our structure condition (1.2). But if we consider functions $h$ such that

$$
h^{\prime \prime}=1 \text { on }[0, \infty)-\bigcup_{i=1}^{\infty} I_{i},
$$

where $I_{i}:=\left[a_{i}-\varepsilon, a_{i}+\varepsilon_{i}\right]$ for an appropriate sequence $a_{i} \rightarrow \infty$ with very small $\varepsilon_{i}$, and if we let $h^{\prime \prime}\left(a_{i}\right)=a_{i}^{\kappa}$ for a suitable power $\kappa$, then (1.8) is violated, whereas the right-hand side of (A3) together with (1.7) can be guaranteed.

Remark 1.3. The regularity problem under the hypotheses (A1)-(A3) was addressed from a different point of view in the paper $\mathrm{ABF}$ : assuming that the local minimizer is also a locally bounded function, we could prove that $\Omega_{0}=\Omega$, replacing (1.7) by the dimensionless condition $\omega<2$.

Remark 1.4. As stated after (1.6), our variational integral also falls into the category of anisotropic energies, and it is possible to deduce Theorem 1.1 from papers on this subject, provided (compare (1.6) and choose $p=2$ ) we know that $q<p \frac{n}{n-2}$ (or a similar inequality) is true. But if we recall the definition of $q$, it is immediate that such a bound for $q$ is too restrictive.

Now we consider the case of higher order variational problems; i.e., we discuss local minima of variational integrals such as

$$
J[w, \Omega]=\int_{\Omega} h\left(\left|\nabla^{k} w\right|\right) d x,
$$

where $k \geq 2$ is a given integer. The symbol $\nabla^{k} w$ stands for the tensor of all $k$ th order (weak) partial derivatives of the function $w$, and $w$ is assumed to be an element of the space $W_{1, \text { loc }}^{k}\left(\Omega ; \mathbb{R}^{M}\right)$. The appropriate modification of Definition 1.1 for the situation at hand is immediate, and we have the following statement.

Theorem 1.2. Assuming (A1)-(A3) and (1.7), let $u \in W_{1, \mathrm{loc}}^{k}\left(\Omega ; \mathbb{R}^{M}\right), M \geq 1, k \geq 2$, denote a local minimizer of the functional $J$ from (1.9). Then there is an open set $\Omega_{0} \subset \Omega$ of full Lebesgue measure such that $u \in C^{k, \nu}\left(\Omega_{0} ; \mathbb{R}^{M}\right)$ for any $0<\nu<1$.

Remark 1.5. In $\mathrm{ApF}$ we proved partial regularity for the local minimizers of anisotropic variational problems of higher order, but, as outlined in Remark 1.4, the result of Theorem 1.2 follows from Theorem 1.1 in $\mathrm{ApF}$ only under very restrictive assumptions on $h$.

Remark 1.6. We note that, with obvious simplifications, our results apply to the case where $\Omega \subset \mathbb{R}^{2}$, which means that then the hypothesis (1.7) becomes superfluous.

The paper is organized as follows. In $\S 2$ we collect some auxiliary results among which Lemma 2.2 is of separate interest, because it contains a statement on the local higher integrability of $\nabla u$ without using the hypothesis (1.7). $§ 3$ is devoted to the proof of Theorem 1.1, which is based on the blow-up technique. In $\S 4$ we sketch how to adjust these arguments to the higher order case described in Theorem 1.2.

\section{§2. Preliminary Results}

Throughout this section we consider a local minimizer $u$ as defined in Definition 1.1 and assume the validity of (A1)-(A3), whereas (1.7) is not required. The following calculations can be made precise by replacing $u, H, I$ by a suitable local regularization $u_{\delta}, H_{\delta}, I_{\delta}, \delta>0$, with exponent $q$, as outlined, e.g., in BF1. 
Lemma 2.1. Let $\Psi:=\int_{0}^{|\nabla u|} \sqrt{\frac{h^{\prime}(t)}{t}} d t$. Then for balls $B_{R}\left(x_{0}\right) \subset \Omega$ and for $0<t<1$ we have

$$
\int_{B_{t R}\left(x_{0}\right)}|\nabla \Psi|^{2} d x \leq c R^{-2}(1-t)^{-2} \int_{B_{R}\left(x_{0}\right)} h(|\nabla u|) d x .
$$

Proof. Let $\eta \in C_{0}^{\infty}(\Omega)$. We have (summation over $\alpha=1, \ldots, n$ )

$$
\begin{aligned}
0 & =\int_{\Omega} \partial_{\alpha}(D H(\nabla u)): \nabla\left(\eta^{2} \partial_{\alpha} u\right) d x \\
& =\int_{\Omega} \partial_{\alpha}(D H(\nabla u)): \partial_{\alpha} \nabla u \eta^{2} d x+\int_{\Omega} \partial_{\alpha}(D H(\nabla u)):\left(\nabla \eta^{2} \otimes \partial_{\alpha} u\right) d x,
\end{aligned}
$$

where ":" is the scalar product of matrices, and " $\otimes$ " denotes the tensor product. We obtain

$$
\int_{\Omega} \partial_{\alpha}(D H(\nabla u)): \partial_{\alpha} \nabla u \eta^{2} d x=\int_{\Omega} D H(\nabla u): \partial_{\alpha}\left(\nabla \eta^{2} \otimes \partial_{\alpha} u\right) d x .
$$

Using the first inequality in (1.5) and observing that $|D H(Z)| \leq h^{\prime}(|Z|)$, we get

$$
\begin{aligned}
& \int_{\Omega} \eta^{2} \frac{h^{\prime}(|\nabla u|)}{|\nabla u|}\left|\nabla^{2} u\right|^{2} d x \\
& \leq \int_{\Omega} h^{\prime}(|\nabla u|)|\nabla u|\left|\nabla^{2} \eta^{2}\right| d x+2 \int_{\Omega} h^{\prime}(\nabla u \mid) \eta|\nabla \eta|\left|\nabla^{2} u\right| d x \\
& \quad \stackrel{\text { (1.4) }}{\leq} \int_{\Omega}\left|\nabla^{2} \eta^{2}\right| h(|\nabla u|) d x \\
& \quad+2 \int_{\Omega}\left(\frac{h^{\prime}(|\nabla u|)}{|\nabla u|}\right)^{1 / 2} \eta\left|\nabla^{2} u\right|\left(h^{\prime}(|\nabla u|)|\nabla u|\right)^{1 / 2}|\nabla \eta| d x .
\end{aligned}
$$

The Young inequality together with another application of (1.4) yields

$$
\int_{\Omega} \eta^{2} \frac{h^{\prime}(|\nabla u|)}{|\nabla u|}\left|\nabla^{2} u\right|^{2} d x \leq c \int_{\Omega}\left[|\nabla \eta|^{2}+\left|\nabla^{2} \eta^{2}\right|\right] h(|\nabla u|) d x .
$$

Since $|\nabla \Psi|^{2} \leq \frac{h^{\prime}(|\nabla u|)}{|\nabla u|}\left|\nabla^{2} u\right|^{2}$, (2.1) follows from (2.2) by specifying $\eta$.

Now we give upper and lower bounds for the function $\Psi$. We have

$$
\begin{aligned}
\Psi & \geq \int_{|\nabla u| / 2}^{|\nabla u|} \sqrt{\frac{h^{\prime}(t)}{t}} d t \geq \frac{|\nabla u|}{2} \sqrt{h^{\prime}(|\nabla u| / 2) /(|\nabla u| / 2)} \\
& =\sqrt{\frac{1}{2}|\nabla u| h^{\prime}(|\nabla u| / 2)} \stackrel{(1.4)}{\geq} \sqrt{h(|\nabla u| / 2)}
\end{aligned}
$$

by the monotonicity of $t \mapsto \frac{h^{\prime}(t)}{t}$. Hence, by (A2),

$$
h(|\nabla u|) \leq c \Psi^{2} .
$$

On the other hand, we clearly have $\Psi \leq|\nabla u| \sqrt{\frac{h^{\prime}(|\nabla u|)}{|\nabla u|}}$, whence

$$
\Psi^{2} \leq \operatorname{ch}(|\nabla u|) .
$$

The right-hand side of (2.4) is in $L_{\text {loc }}^{1}(\Omega)$; together with (2.1), this implies that $\Psi \in$ $W_{2, \text { loc }}^{1}(\Omega)$. By Sobolev's theorem in combination with (2.3), we obtain the following statement.

Lemma 2.2. We have $h(|\nabla u|)^{\frac{n}{n-2}} \in L_{\mathrm{loc}}^{1}(\Omega)$. 
Next we recall the Caccioppoli-type inequality (see, e.g., BF1]),

$$
\int_{\Omega} \eta^{2} D^{2} H(\nabla u)\left(\partial_{\alpha} \nabla u, \partial_{\alpha} \nabla u\right) d x \leq c \int_{\Omega}\left|D^{2} H(\nabla u)\right||\nabla \eta|^{2}|\nabla u-Q|^{2} d x,
$$

valid for $\eta \in C_{0}^{\infty}(\Omega)$ and $Q \in \mathbb{R}^{n M}$. This inequality implies that

$$
\int_{\Omega} \eta^{2} \frac{h^{\prime}(|\nabla u|)}{|\nabla u|}\left|\nabla^{2} u\right|^{2} d x \leq c \int_{\Omega}|\nabla \eta|^{2}\left(1+|\nabla u|^{2}\right)^{\frac{\omega}{2}} \frac{h^{\prime}(|\nabla u|)}{|\nabla u|}|\nabla u-Q|^{2} d x .
$$

Note that the integrands on the right in (2.5) and (2.6) behave like $|\nabla u|^{\omega} h(|\nabla u|)$, and by Lemma 2.2, their local integrability will follow if condition (1.7) is valid.

\section{§3. Proof of Theorem 1.1 VIA Blow-UP}

From now on we assume that the conditions of Theorem 1.1 are fulfilled. Let $u$ denote a local $I$-minimizer and suppose that $\omega>0$ in A3). (Otherwise the claim of Theorem 1.1 follows with $\Omega_{0}=\Omega$ from the results of $\mathrm{MP}$.) Next, let

$$
\widetilde{h}(t):=t^{\omega} h(t), \quad t \geq 0,
$$

and observe that $\widetilde{h}$ is an $N$-function. From (1.7) and Lemma 2.2, it follows that $u \in$ $W_{\widetilde{h}, \text { loc }}^{1}\left(\Omega ; \mathbb{R}^{M}\right)$, so that the excess-function

$$
E(x, r):=f_{B_{r}(x)}\left|\nabla u-(\nabla u)_{x, r}\right|^{2} d y+f_{B_{r}(x)} \tilde{h}\left(\left|\nabla u-(\nabla u)_{x, r}\right|\right) d y
$$

for balls $B_{r}(x) \Subset \Omega$ is well defined. Here and in what follows, $f_{\ldots} f,(f) \ldots$ denote the mean value of a function $f$.

Lemma 3.1. Fix $L>0$ and a subdomain $\Omega^{\prime} \Subset \Omega$. Then there is a constant $C_{*}(L)$ such that for every $\tau \in(0,1)$ there is a number $\varepsilon=\varepsilon(L, \tau)$ with the following property: if $B_{r}(x) \subset \Omega^{\prime}$ and

$$
\left|(\nabla u)_{x, r}\right| \leq L, \quad E(x, r) \leq \varepsilon
$$

then

$$
E(x, \tau r) \leq C_{*}(L) \tau^{2} E(x, r) .
$$

Once having established Lemma 3.1, it is standard (see, e.g., Giaquinta's textbook Gi]) to prove the desired partial regularity result. It turns out that the regular set $\Omega_{0}$ is given by

$$
\Omega_{0}=\left\{x \in \Omega: \sup _{r>0}\left|(\nabla u)_{x, r}\right|<\infty \text { and } \liminf _{r \downarrow 0} E(x, r)=0\right\} ;
$$

i.e., Lemma 3.1 shows that the set on the right-hand side is open and $\nabla u \in C^{0, \alpha}$ there for any $0<\alpha<1$. Obviously, this set is of full Lebesgue measure.

We divide the proof of Lemma 3.1 into several steps.

Step 1. Scaling. We argue by contradiction. Let $L>0$ and choose $C_{*}=C_{*}(L)$ as outlined at Step 2. Then, for some $\tau \in(0,1)$, there is a sequence of balls $B_{r_{m}}\left(x_{m}\right) \Subset \Omega^{\prime}$ such that

$$
\begin{aligned}
& \left|(\nabla u)_{x_{m}, r_{m}}\right| \leq L, \quad E\left(x_{m}, r_{m}\right)=: \lambda_{m}^{2} \rightarrow 0 \text { as } m \rightarrow \infty, \\
& E\left(x_{m}, \tau r_{m}\right)>C_{*} \tau^{2} \lambda_{m}^{2} .
\end{aligned}
$$

Letting $a_{m}:=(u)_{x_{m}, r_{m}}, A_{m}:=(\nabla u)_{x_{m}, r_{m}}$, for $z \in B_{1}:=B_{1}(0)$ we define

$$
u_{m}(z):=\frac{1}{\lambda_{m} r_{m}}\left[u\left(x_{m}+r_{m} z\right)-a_{m}-r_{m} A_{m} z\right] .
$$


Now, by (3.3),

$$
\left|A_{m}\right| \leq L, \quad f_{B_{1}}\left|\nabla u_{m}\right|^{2} d z+\lambda_{m}^{-2} f_{B_{1}} \tilde{h}\left(\lambda_{m}\left|\nabla u_{m}\right|\right) d z=1 .
$$

On the other hand, after scaling, (3.4) reads

$$
f_{B_{\tau}}\left|\nabla u_{m}-\left(\nabla u_{m}\right)_{0, \tau}\right|^{2} d z+\lambda_{m}^{-2} f_{B_{\tau}} \tilde{h}\left(\lambda_{m}\left|\nabla u_{m}-\left(\nabla u_{m}\right)_{0, \tau}\right|\right) d z>C_{*} \tau^{2} .
$$

Passing to suitable subsequences, from (3.5) we obtain

$$
\begin{aligned}
A_{m} \rightarrow: A, u_{m} \rightarrow: \bar{u} & \text { in } W_{2}^{1}\left(B_{1} ; \mathbb{R}^{M}\right), \\
\lambda_{m} \nabla u_{m} \rightarrow 0 & \text { in } L^{2}\left(B_{1} ; \mathbb{R}^{n M}\right) \text { and a.e. }
\end{aligned}
$$

where, obviously, $(\bar{u})_{0,1}=0,(\nabla \bar{u})_{0,1}=0$.

Step 2. Limit equation. For any $\varphi \in C_{0}^{\infty}\left(B_{1} ; \mathbb{R}^{M}\right)$ the Euler equation satisfied by $u$ implies after scaling that

$$
\begin{aligned}
\int_{B_{1}} & D^{2} H\left(A_{m}\right)\left(\nabla u_{m}, \nabla \varphi\right) d z \\
& =-\int_{B_{1}} \int_{0}^{1}\left[D^{2} H\left(Z_{m}\right)-D^{2} H\left(A_{m}\right)\right]\left(\nabla u_{m}, \nabla \varphi\right) d s d z,
\end{aligned}
$$

where we have abbreviated

$$
Z_{m}:=Z_{m}(s, z):=A_{m}+s \lambda_{m} \nabla u_{m}(z) .
$$

The first line of (3.7) shows that

$$
\text { the left-hand side of }\left(\underline{3.8)} \stackrel{m \rightarrow \infty}{\longrightarrow} \int_{B_{1}} D^{2} H(A)(\nabla \bar{u}, \nabla \varphi) d z\right. \text {. }
$$

For discussing the right-hand side of (3.8), we let $\varepsilon>0$ and choose $\delta=\delta(\varepsilon)$ such that

$$
\int_{A}|\nabla \varphi|^{2} d z \leq \varepsilon
$$

for any measurable subset $A$ of $B_{1}$ with $\mathcal{L}^{n}(A) \leq \delta$. The second line of (3.7) gives the existence of $S \subset B_{1}$ such that $\mathcal{L}^{n}\left(B_{1}-S\right) \leq \delta$ and

$$
\lambda_{m} \nabla u_{m} \rightrightarrows 0 \text { on } S \text {. }
$$

Using (3.11) and also (3.5), we immediately see that

$$
\begin{aligned}
& \left|\int_{S} \int_{0}^{1}\left[D^{2} H\left(Z_{m}\right)-D^{2} H\left(A_{m}\right)\right]\left(\nabla u_{m}, \nabla \varphi\right) d s d z\right| \\
& \quad \leq \sup _{S \times[0,1]}|[\ldots]|\left(\int_{B_{1}}\left|\nabla u_{m}\right|^{2} d z\right)^{1 / 2}\left(\int_{B_{1}}|\nabla \varphi|^{2} d z\right)^{1 / 2} \rightarrow 0
\end{aligned}
$$


as $m \rightarrow \infty$. At the same time, we use (1.5) to show that

$$
\begin{aligned}
T & :=\left|\int_{B_{1}-S} \int_{0}^{1}[\ldots]\left(\nabla u_{m}, \nabla \varphi\right) d s d z\right| \\
& \leq c \int_{B_{1}-S} \int_{0}^{1}\left\{1+\left|D^{2} H\left(Z_{m}\right)\right|\right\}\left|\nabla u_{m}\right||\nabla \varphi| d s d z \\
& \leq c \int_{B_{1}-S} \int_{0}^{1}\left\{1+\left(1+\left|Z_{m}\right|^{2}\right)^{\frac{\omega}{2}} \frac{h^{\prime}\left(\left|Z_{m}\right|\right)}{\left|Z_{m}\right|}\right\}\left|\nabla u_{m}\right||\nabla \varphi| d s d z \\
& =c\left\{\int_{\left(B_{1}-S\right) \cap M_{1}} \int_{0}^{1} \ldots d s d z+\int_{\left(B_{1}-S\right) \cap M_{2}} \int_{0}^{1} \ldots d s d z\right\} \\
& =: c\left\{T_{1}+T_{2}\right\},
\end{aligned}
$$

where we have abbreviated

$$
M_{1}:=\left[\lambda_{m}\left|\nabla u_{m}\right| \leq K\right], \quad M_{2}:=\left[\lambda_{m}\left|\nabla u_{m}\right|>K\right]
$$

for a sufficiently large number $K$. On $M_{1}$ we have

$$
\left|Z_{m}\right| \leq \sup _{m}\left|A_{m}\right|+K \leq L+K=: k
$$

so that

$$
\left(1+\left|Z_{m}\right|^{2}\right)^{\frac{\omega}{2}} \frac{h^{\prime}\left(\left|Z_{m}\right|\right)}{\left|Z_{m}\right|} \leq c(K) \frac{h^{\prime}(k)}{k} \stackrel{\text { A3 } 3}{\leq} c(K) h^{\prime \prime}(k),
$$

and, in conclusion,

$$
\begin{aligned}
T_{1} & \leq c(K) \int_{B_{1}-S}\left|\nabla u_{m}\right||\nabla \varphi| d z \\
& \leq c(K)\left(\int_{B_{1}-S}\left|\nabla u_{m}\right|^{2} d z\right)^{1 / 2}\left(\int_{B_{1}-S}|\nabla \varphi|^{2} d z\right)^{1 / 2} \\
& \quad \leq c(K) \sqrt{\varepsilon} .
\end{aligned}
$$

For $T_{2}$ we obtain (assuming $\left|Z_{m}\right| \leq 2 \lambda_{m}\left|\nabla u_{m}\right|$ on $M_{2}$ )

$$
\begin{aligned}
T_{2} \leq & c \int_{\left(B_{1}-S\right) \cap M_{2}}\left|\nabla u_{m}\right||\nabla \varphi| d z \\
& +c \int_{\left(B_{1}-S\right) \cap M_{2}}\left(\lambda_{m}\left|\nabla u_{m}\right|\right)^{\omega} \frac{h^{\prime}\left(2 \lambda_{m}\left|\nabla u_{m}\right|\right)}{2 \lambda_{m}\left|\nabla u_{m}\right|}\left|\nabla u_{m}\right||\nabla \varphi| d z,
\end{aligned}
$$

and, as before, the first integral on the right-hand side is bounded by $c \sqrt{\varepsilon}$. Using (1.4) and (A2), we get

$$
T_{2} \leq c \sqrt{\varepsilon}+c \lambda_{m}^{-1} \int_{\left(B_{1}-S\right) \cap M_{2}}\left(\lambda_{m}\left|\nabla u_{m}\right|\right)^{\omega-1} h\left(\lambda_{m}\left|\nabla u_{m}\right|\right)|\nabla \varphi| d z .
$$

Recalling the definition of $\tilde{h}$, we see that

$$
\omega t^{\omega-1} h(t) \leq \widetilde{h}^{\prime}(t)
$$

whence

$$
T_{2} \leq c \sqrt{\varepsilon}+c \lambda_{m}^{-1} \int_{\left(B_{1}-S\right) \cap M_{2}} \tilde{h}^{\prime}\left(\lambda_{m}\left|\nabla u_{m}\right|\right)|\nabla \varphi| d z
$$


and it remains to discuss the integral on the right-hand side of (3.14). For this, we put $g_{\tau}(t):=\tau \widetilde{h}^{*}(t)$, for $\tau>0$, where $\widetilde{h}^{*}$ is the conjugate function of $\widetilde{h}$, and use Young's inequality in the form

$$
\alpha \beta \leq g_{\tau}(\alpha)+g_{\tau}^{*}(\beta),
$$

for numbers $\alpha, \beta \geq 0$. With $\alpha:=\widetilde{h}^{\prime}\left(\lambda_{m}\left|\nabla u_{m}\right|\right), \beta:=|\nabla \varphi|$, we obtain

$$
\begin{aligned}
\widetilde{h}^{\prime}\left(\lambda_{m}\left|\nabla u_{m}\right|\right)|\nabla \varphi| & \leq \tau \widetilde{h}^{*}\left(\widetilde{h}^{\prime}\left(\lambda_{m}\left|\nabla u_{m}\right|\right)\right)+g_{\tau}^{*}(|\nabla \varphi|) \\
& =\tau\left[\lambda_{m}\left|\nabla u_{m}\right| \widetilde{h}^{\prime}\left(\lambda_{m}\left|\nabla u_{m}\right|\right)-\widetilde{h}\left(\lambda_{m}\left|\nabla u_{m}\right|\right)\right]+g_{\tau}^{*}(|\nabla \varphi|) \\
& \leq c \tau \widetilde{h}\left(\lambda_{m}\left|\nabla u_{m}\right|\right)+g_{\tau}^{*}(|\nabla \varphi|), \\
g_{\tau}^{*}(|\nabla \varphi|) & =\sup _{\gamma \geq 0}\left[\beta \gamma-\tau \widetilde{h}^{*}(\gamma)\right] \\
& =\tau \sup _{\gamma \geq 0}\left[\frac{\beta}{\tau} \gamma-\widetilde{h}^{*}(\gamma)\right]=\tau \widetilde{h}(\beta / \tau)=\tau \widetilde{h}(|\nabla \varphi| / \tau) .
\end{aligned}
$$

This gives

$$
\begin{aligned}
\xi_{m} & :=\lambda_{m}^{-1} \int_{\left(B_{1}-S\right) \cap M_{2}} \tilde{h}^{\prime}\left(\lambda_{m}\left|\nabla u_{m}\right|\right)|\nabla \varphi| d z \\
& \leq c \lambda_{m}^{-1} \tau \int_{B_{1}} \tilde{h}\left(\lambda_{m}\left|\nabla u_{m}\right|\right) d z+c \lambda_{m}^{-1} \tau \int_{B_{1}} \tilde{h}\left(\frac{1}{\tau}|\nabla \varphi|\right) d z
\end{aligned}
$$

Now, let $\tau:=\lambda_{m}^{\delta-1}$ for a small $\delta>0$. Then

$$
\xi_{m} \leq c\left[\lambda_{m}^{\delta-2} \int_{B_{1}} \tilde{h}\left(\lambda_{m}\left|\nabla u_{m}\right|\right) d z+\lambda_{m}^{\delta-2} \int_{B_{1}} \tilde{h}\left(\lambda_{m}^{1-\delta}|\nabla \varphi|\right) d z\right] .
$$

The definition of $\tilde{h}$ shows that, for any $\ell>0$,

$$
\widetilde{h}(t) \leq \operatorname{const}(\ell) t^{2+\omega}, \quad 0 \leq t \leq \ell,
$$

where const $(\ell)$ depends on the value of $\ell$. Here we choose $\ell:=\|\nabla \varphi\|_{\infty}$, obtaining

$$
\lambda_{m}^{\delta-2} \int_{B_{1}} \tilde{h}\left(\lambda_{m}^{1-\delta}|\nabla \varphi|\right) d z \leq c\left(\|\nabla \varphi\|_{\infty}\right) \lambda_{m}^{\delta-2+(1-\delta)(2+\omega)} .
$$

Since $\omega$ is positive, it is possible to choose $\delta>0$ such that

$$
\lambda_{m}^{\delta-2} \int_{B_{1}} \tilde{h}\left(\lambda_{m}^{1-\delta}|\nabla \varphi|\right) d z \rightarrow 0, \quad m \rightarrow \infty
$$

Since, by (3.5),

$$
\lambda_{m}^{\delta-2} \int_{B_{1}} \widetilde{h}\left(\lambda_{m}\left|\nabla u_{m}\right|\right) d z \rightarrow 0, \quad m \rightarrow \infty,
$$

it follows that $\lim _{m \rightarrow \infty} \xi_{m}=0$. Combining this fact with estimates (3.12)-(3.14), we obtain

$$
\limsup _{m \rightarrow \infty} \mid \text { right-hand side of }(3.8) \mid \leq c \sqrt{\varepsilon} \text {. }
$$

Now $\varepsilon$ can be chosen as small as we want; hence, (3.8) and (3.9) together with (3.15) lead to the elliptic system with constant coefficients

$$
\int_{B_{1}} D^{2} H(A)(\nabla \bar{u}, \nabla \varphi) d z=0
$$


satisfied by $\bar{u}$. In accordance with $\mathrm{Gi}$, $\bar{u}$ is of class $C^{\infty}\left(B_{1} ; \mathbb{R}^{M}\right)$, and we have the Campanato estimate

$$
f_{B_{\tau}}\left|\nabla \bar{u}-(\nabla \bar{u})_{0, \tau}\right|^{2} d z \leq C^{*} \tau^{2} f_{B_{1}}\left|\nabla \bar{u}-(\nabla \bar{u})_{0,1}\right|^{2} d z
$$

with a constant $C^{*}=C^{*}(L)$. Observing that $f_{B_{1}}|\nabla \bar{u}|^{2} d z \leq 1$ (see (3.5)) and recalling that $(\nabla \bar{u})_{0,1}=0$, we get

$$
f_{B_{\tau}}\left|\nabla \bar{u}-(\nabla \bar{u})_{0, \tau}\right|^{2} d z \leq C^{*} \tau^{2} .
$$

Letting $C_{*}:=2 C^{*}$, we see that (3.16) contradicts (3.6) as soon as we can show that

$$
\begin{aligned}
& \nabla u_{m} \rightarrow \nabla \bar{u} \text { in } L_{\mathrm{loc}}^{2}\left(B_{1} ; \mathbb{R}^{n M}\right), \\
& \lambda_{m}^{-2} f_{B_{r}} \tilde{h}\left(\lambda_{m}\left|\nabla u_{m}\right|\right) d z \rightarrow 0, \quad r<1 .
\end{aligned}
$$

In fact, (3.17) implies the convergence of the first integral on the left-hand side of (3.6) to the integral on the left-hand side of (3.16). Next, we have

$$
\begin{aligned}
& \lambda_{m}^{-2} f_{B_{\tau}} \tilde{h}\left(\lambda_{m}\left|\nabla u_{m}-\left(\nabla u_{m}\right)_{0, \tau}\right|\right) d z \\
& \quad \leq c\left[\lambda_{m}^{-2} f_{B_{\tau}} \tilde{h}\left(\lambda_{m}\left|\nabla u_{m}\right|\right) d z+\lambda_{m}^{-2} \widetilde{h}\left(\lambda_{m}\left|\left(\nabla u_{m}\right)_{0, \tau}\right|\right)\right],
\end{aligned}
$$

which follows from the convexity and the $(\Delta 2)$-property of $\widetilde{h}$. By (3.18), the first term on the right-hand side of the above inequality vanishes as $m \rightarrow \infty$, and (3.17) yields $\left|\left(\nabla u_{m}\right)_{0, \tau}\right| \longrightarrow\left|(\nabla \bar{u})_{0, \tau}\right|$ as $m \longrightarrow \infty$; hence, $\lambda_{m}^{-2} \widetilde{h}\left(\lambda_{m}\left|\left(\nabla u_{m}\right)_{0, \tau}\right|\right)$ behaves like $\lambda_{m}^{-2} \lambda_{m}^{2+\omega} \stackrel{m \rightarrow \infty}{\longrightarrow} 0$, and therefore, the second term on the left-hand side of (3.6) vanishes as $m \rightarrow \infty$.

Step 3. Proof of (3.17) and (3.18). We return to (2.6), observe that $\frac{h^{\prime}(t)}{t} \geq h^{\prime \prime}(0)$, and choose $Q=A_{m}$. After scaling (for a suitable choice of $\eta$ ), we obtain

$$
\int_{B_{t}}\left|\nabla^{2} u_{m}\right| d z \leq C(1-t)^{-2} \int_{B_{1}}\left|D^{2} H\left(\lambda_{m} \nabla u_{m}+A_{m}\right)\right|\left|\nabla u_{m}\right|^{2} d z
$$

for $0<t<1$. On $\left[\lambda_{m}\left|\nabla u_{m}\right| \leq K\right]$ we have

$$
\left|D^{2} H\left(A_{m}+\lambda_{m} \nabla u_{m}\right)\right|\left|\nabla u_{m}\right|^{2} \leq c(K)\left|\nabla u_{m}\right|^{2},
$$

whereas on $\left[\lambda_{m}\left|\nabla u_{m}\right| \geq K\right]$ with $K$ sufficiently large we have

$$
\begin{aligned}
\left|D^{2} H\left(\lambda_{m} \nabla u_{m}+A_{m}\right)\right|\left|\nabla u_{m}\right|^{2} & \leq c(K)\left[1+\left(\lambda_{m}\left|\nabla u_{m}\right|\right)^{\omega} \frac{h^{\prime}\left(\lambda_{m}\left|\nabla u_{m}\right|\right)}{\lambda_{m}\left|\nabla u_{m}\right|}\right]\left|\nabla u_{m}\right|^{2} \\
& \leq c(K)\left[\left|\nabla u_{m}\right|^{2}+\lambda_{m}^{-2} \widetilde{h}\left(\lambda_{m}\left|\nabla u_{m}\right|\right)\right] .
\end{aligned}
$$

Therefore, (3.19) implies that

$$
\int_{B_{t}}\left|\nabla^{2} u_{m}\right|^{2} d z \leq c(1-t)^{-2}\left[\int_{B_{1}}\left|\nabla u_{m}\right|^{2} d z+\lambda_{m}^{-2} \int_{B_{1}} \tilde{h}\left(\lambda_{m}\left|\nabla u_{m}\right|\right) d z\right],
$$

and, by (3.5), the right-hand side of (3.20) is bounded. Together with (3.7), this proves (3.17). Let $c(t)$ denote a bound for the right-hand side of (3.20). Then, for $t \in(0,1)$, another application of (2.6) yields

$$
\int_{B_{t}} \frac{h^{\prime}\left(\left|\lambda_{m} \nabla u_{m}+A_{m}\right|\right)}{\left|\lambda_{m} \nabla u_{m}+A_{m}\right|}\left|\nabla^{2} u_{m}\right|^{2} d z \leq c(t)
$$


We introduce the auxiliary functions

$$
\Psi_{m}:=\frac{1}{\lambda_{m}}\left\{\int_{0}^{\left|\lambda_{m} \nabla u_{m}+A_{m}\right|} \sqrt{\frac{h^{\prime}(t)}{t}} d t-\int_{0}^{\left|A_{m}\right|} \sqrt{\frac{h^{\prime}(t)}{t}} d t\right\}
$$

and observe that, by (3.21),

$$
\int_{B_{t}}\left|\nabla \Psi_{m}\right|^{2} d z \leq c(t)
$$

On $\left[\lambda_{m}\left|\nabla u_{m}\right| \leq K\right]$ we have

$$
\left|\Psi_{m}\right|=\frac{1}{\lambda_{m}}\left|\int_{\left|A_{m}\right|}^{\left|A_{m}+\lambda_{m} \nabla u_{m}\right|} \sqrt{\frac{h^{\prime}(t)}{t}} d t\right| \leq \frac{1}{\lambda_{m}}|| A_{m}+\lambda_{m} \nabla u_{m}|-| A_{m}|| \sqrt{\frac{h^{\prime}(k)}{k}},
$$

where $k:=K+\sup _{m}\left|A_{m}\right|$. Therefore, we get

$$
\left|\Psi_{m}\right| \leq \sqrt{\frac{h^{\prime}(k)}{k}}\left|\nabla u_{m}\right|
$$

provided that $\lambda_{m}\left|\nabla u_{m}\right| \leq K$. For $K$ sufficiently large, we can assume that

$$
\left|A_{m}+\lambda_{m} \nabla u_{m}\right| \leq 2 \lambda_{m}\left|\nabla u_{m}\right|
$$

on $\left[\lambda_{m}\left|\nabla u_{m}\right| \geq K\right]$, whence

$$
\begin{aligned}
\left|\Psi_{m}\right| \leq c \frac{1}{\lambda_{m}} \lambda_{m}\left|\nabla u_{m}\right| \sqrt{\frac{h^{\prime}\left(2 \lambda_{m}\left|\nabla u_{m}\right|\right)}{2 \lambda_{m}\left|\nabla u_{m}\right|}} & \leq c \lambda_{m}^{-1} h\left(\lambda_{m}\left|\nabla u_{m}\right|\right)^{1 / 2} \\
& \leq c \lambda_{m}^{-1} \widetilde{h}\left(\lambda_{m}\left|\nabla u_{m}\right|\right)^{1 / 2}
\end{aligned}
$$

if $\lambda_{m}\left|\nabla u_{m}\right| \geq K$. Recalling the bounds from (3.5), we can show that

$$
\int_{B_{1}} \Psi_{m}^{2} d z \leq c<\infty
$$

and together with (3.22) this implies $(0<t<1)$ that

$$
\left\|\Psi_{m}\right\|_{W_{2}^{1}\left(B_{t}\right)} \leq c(t)<\infty .
$$

Let $A_{K}(t):=B_{t} \cap\left[\lambda_{m}\left|\nabla u_{m}\right| \leq K\right]$. We use $\tilde{h}(t) \leq c_{K} t^{2+\omega}=: c_{K} t^{s}$ for $t \leq K$ to get

$$
\begin{aligned}
\int_{A_{K}(t)} & \lambda_{m}^{-2} \widetilde{h}\left(\lambda_{m}\left|\nabla u_{m}\right|\right) d z \\
\leq & c\left[\lambda_{m}^{s-2} \int_{A_{K}(t)}\left|\nabla u_{m}-\nabla \bar{u}\right|^{s} d z+\lambda_{m}^{s-2} \int_{A_{K}(t)}|\nabla \bar{u}|^{s} d z\right] \\
\leq & c\left[\lambda_{m}^{s-2} \int_{A_{k}(t)}\left\{\left|\nabla u_{m}\right|^{s-2}+|\nabla \bar{u}|^{s-2}\right\}\left|\nabla u_{m}-\nabla \bar{u}\right|^{2} d z+\lambda_{m}^{s-2} \int_{A_{k}(t)}|\nabla \bar{u}|^{s} d z\right] .
\end{aligned}
$$

Using the local boundedness of $\nabla \bar{u}$, and also (3.17), we see that

$$
\lambda_{m}^{-2} \int_{A_{K}(t)} \tilde{h}\left(\lambda_{m}\left|\nabla u_{m}\right|\right) d z \longrightarrow 0, \quad m \rightarrow \infty .
$$

Now consider the set $\widetilde{A}_{K}(t):=B_{t} \cap\left[\lambda_{m}\left|\nabla u_{m}\right| \geq K\right]$, on which the following estimates are valid:

$$
\begin{aligned}
\Psi_{m} & =\frac{1}{\lambda_{m}} \int_{\left|A_{m}\right|}^{\left|\lambda_{m} \nabla u_{m}+A_{m}\right|} \sqrt{\frac{h^{\prime}(t)}{t}} d t \geq \frac{1}{\lambda_{m}} \int_{\frac{1}{2} \lambda_{m}\left|\nabla u_{m}\right|}^{\frac{2}{3} \lambda_{m}\left|\nabla u_{m}\right|} \sqrt{\frac{h^{\prime}(t)}{t}} d t \\
& \geq c \frac{1}{\lambda_{m}} \lambda_{m}\left|\nabla u_{m}\right| \sqrt{\frac{h^{\prime}\left(\lambda_{m}\left|\nabla u_{m}\right| / 2\right)}{\frac{1}{2} \lambda_{m}\left|\nabla u_{m}\right|}} \geq \frac{c}{\lambda_{m}} \sqrt{h\left(\lambda_{m}\left|\nabla u_{m}\right|\right)} .
\end{aligned}
$$


This gives

$$
\Psi_{m}^{2} \geq c \lambda_{m}^{-2} h\left(\lambda_{m}\left|\nabla u_{m}\right|\right) \text { on } \widetilde{A}_{K}(t)
$$

Therefore,

$$
\begin{aligned}
\lambda_{m}^{-2} & \int_{\widetilde{A}_{K}(t)} \tilde{h}\left(\lambda_{m}\left|\nabla u_{m}\right|\right) d z=\lambda_{m}^{-2} \int_{\widetilde{A}_{K}(t)}\left(\lambda_{m}\left|\nabla u_{m}\right|\right)^{\omega} h\left(\lambda_{m}\left|\nabla u_{m}\right|\right) d z \\
& \quad \leq c \int_{\tilde{A}_{K}(t)}\left(\lambda_{m}\left|\nabla u_{m}\right|\right)^{\omega} \Psi_{m}^{2} d z \\
& \leq c\left(\int_{B_{t}} \Psi_{m}^{\frac{2 n}{n-2}} d z\right)^{1-2 / n}\left(\int_{\widetilde{A}_{K}(t)}\left(\lambda_{m}\left|\nabla u_{m}\right|\right)^{\omega \frac{n}{2}} d z\right)^{2 / n} \\
& \stackrel{(3.23)}{\leq} c(t)\left(\int_{\widetilde{A}_{K}(t)}\left(\lambda_{m}\left|\nabla u_{m}\right|\right)^{\omega \frac{n}{2}} d z\right)^{2 / n} \\
& \stackrel{(1.7)}{\leq} c(t)\left[\mathcal{L}^{n}\left(\widetilde{A}_{K}(t)\right)+\int_{\widetilde{A}_{K}(t)} h\left(\lambda_{m}\left|\nabla u_{m}\right|\right)^{\frac{n}{n-2}} d z\right] .
\end{aligned}
$$

The second line of (3.7) shows that $\mathcal{L}^{n}\left(\widetilde{A}_{K}(t)\right) \rightarrow 0$ as $m \rightarrow \infty$; moreover, we have

$$
\begin{aligned}
\int_{\widetilde{A}_{K}(t)} h\left(\lambda_{m}\left|\nabla u_{m}\right|\right)^{\frac{n}{n-2}} d z & \stackrel{(3.25)}{\leq} c \lambda_{m}^{\frac{2 n}{n-2}} \int_{B_{t}} \Psi_{m}^{\frac{2 n}{n-2}} d z \\
& \stackrel{(3.23)}{\leq} c(t) \lambda_{m}^{\frac{2 n}{n-2}} \longrightarrow 0, \quad m \rightarrow \infty,
\end{aligned}
$$

whence $\int_{\widetilde{A}_{K}(t)} \lambda_{m}^{-2} \widetilde{h}\left(\lambda_{m}\left|\nabla u_{m}\right|\right) d z \longrightarrow 0, m \rightarrow \infty$. Combined with (3.24), this proves (3.18), completing the proof of Lemma 3.1 and thereby of Theorem 1.1.

\section{§4. Sketch of the Proof of Theorem 1.2}

Clearly, we can restrict ourselves to the case where $k=2$ and $M=1$. Then we introduce a local regularization, as done in $\S 2$ of $\mathrm{ApF}$, and drop the approximation parameter.

Lemma 4.1. The function

$$
\Psi:=\int_{0}^{\left|\nabla^{2} u\right|} \sqrt{\frac{h^{\prime}(t)}{t}} d t
$$

is of class $W_{2, \mathrm{loc}}^{1}(\Omega)$ (uniformly with respect to the approximation).

A complete proof of Lemma 4.1 is presented in [Fu1, Step 2 of the proof of Theorem 1]. Lemma 2.2 should be replaced by the following statement.

Lemma 4.2. The function $h\left(\left|\nabla^{2} u\right|\right)^{\frac{n}{n-2}}$ is in the space $L_{\text {loc }}^{1}(\Omega)$ (uniformly with respect to the regularization).

A version of the Caccioppoli inequality (2.5) valid for the higher order case was established in (2.11) of $\mathrm{ApF}$. During the blow-up procedure we need the following adjustments: now we let

$$
E(x, r):=f_{B_{r}(x)}\left|\nabla^{2} u-\left(\nabla^{2} u\right)_{x, r}\right|^{2} d y+f_{B_{r}(x)} \tilde{h}\left(\left|\nabla^{2} u-\left(\nabla^{2} u\right)_{x, r}\right|\right) d y
$$


and require that $\left|\left(\nabla^{2} u\right)_{x, r}\right| \leq L$ in (3.1). We define $a_{m}, A_{m}$ as on the line following (3.4), and put $\Theta_{m}:=\left(\nabla^{2} u\right)_{x_{m}, r_{m}}$ and

$$
\begin{aligned}
\widehat{u}_{m}(z):=\frac{1}{\lambda_{m} r_{m}^{2}}\left[u_{m}\left(x_{m}+r_{m} z\right)-a_{m}-r_{m} A_{m} z\right. & \\
& \left.-\frac{1}{2} r_{m}^{2} \Theta_{m}(z, z)+\frac{1}{2} r_{m}^{2} f_{B_{1}} \Theta_{m}(\tilde{z}, \widetilde{z}) d \tilde{z}\right], \quad z \in B_{1} .
\end{aligned}
$$

Formula (3.7) must be replaced by

$$
\left\{\begin{array}{l}
\Theta_{m} \rightarrow: \Theta, \widehat{u}_{m} \rightarrow: \widehat{u} \text { in } W_{2}^{2}\left(B_{1}\right) \\
\lambda_{m} \nabla^{2} \widehat{u}_{m} \rightarrow 0 \text { in } L^{2}\left(B_{1} ; \mathbb{R}^{n \times n}\right) \text { and a.e. }
\end{array}\right.
$$

Let $H(Z):=h(|Z|)$. Along the lines of Step 2 in $\S 3$, from (4.1) we obtain the limit equation

$$
\int_{B_{1}} D^{2} H(\Theta)\left(\nabla^{2} \widehat{u}, \nabla^{2} \varphi\right) d z=0
$$

valid for all $\varphi \in C_{0}^{\infty}\left(B_{1}\right)$, and to (4.2) we can apply the Campanato-type estimate (3.11) of $\mathrm{ApF}$, by the way fixing the value of $C_{*}$. Finally, we prove (see (3.17) and (3.18) )

$$
\left\{\begin{array}{l}
\nabla^{2} \widehat{u}_{m} \rightarrow \nabla^{2} \widehat{u} \text { in } L_{\text {loc }}^{2}\left(B_{1} ; \mathbb{R}^{n \times n}\right), \\
\lambda_{m}^{-2} f_{B_{\tau}} \tilde{h}\left(\lambda_{m}\left|\nabla^{2} \widehat{u}_{m}\right|\right) d z \rightarrow 0, \quad r<1,
\end{array}\right.
$$

which is done exactly as at Step 3 of $\S 3$. But, as before, (4.3) will lead to the desired contradiction, thus proving Theorem 1.2.

\section{REFERENCES}

[Ad] R. A. Adams, Sobolev spaces, Pure Appl. Math., vol. 65, Acad. Press, New York-London, 1975. MR0450957 (56:9247)

[AM] E. Acerbi and G. Mingione, Regularity results for stationary electro-rheological fluids, Arch. Rational Mech. Anal. 164 (2002), 213-259. MR.1930392 (2003g:35020)

[ABF] D. Apushkinskaya, M. Bildhauer, and M. Fuchs, Interior gradient bounds for local minimizers of variational integrals under nonstandard growth conditions (to appear in J. Math. Sci. 164 (2010), no. 3).

[AF] E. Acerbi and N. Fusco, Partial regularity under anisotropic $(p, q)$ growth conditions, J. Differential Equations 107 (1994), 46-67. MR:1260848 (95c:49059)

$[\mathrm{ApF}] \quad$ D. Apushkinskaya and M. Fuchs, Partial regularity for higher order variational problems under anisotropic growth conditions, Ann. Acad. Sci. Fenn. Math. 32 (2007), 199-214. MR2297886 (2008f:49041)

[BF1] M. Bildhauer and M. Fuchs, Partial regularity for variational integrals with $(s, \mu, q)$-growth, Calc. Var. Partial Differential Equations 13 (2001), 537-560. MR.1867941 (2002h:49057)

[BF2] _ Partial regularity for local minimizers of splitting-type variational integrals, Asymptot. Anal. 55 (2007), 33-47. MR.2373805 (2008k:49083)

[ELM1] L. Esposito, F. Leonetti, and G. Mingione, Regularity for minimizers of functionals with $p$ q growth, NoDEA Nonlinear Differential Equations Appl. 6 (1999), 133-148. MR1694803 (2000h:49056)

[ELM2] , Regularity results for minimizers of irregular integrals with $p$ - $q$ growth, Forum Math. 14 (2002), 245-272. MR 1880913 (2003a:49048)

[Fu1] M. Fuchs, Minimization of energies related to the plate problem, Math. Methods Appl. Sci. 32 (2009), 773-782. MR2507931

[Fu2] _ A note on non-uniformly elliptic Stokes-type systems in two variables, JMFM (to appear).

[Gi] M. Giaquinta, Multiple integrals in the calculus of variations and nonlinear elliptic systems, Ann. of Math. Stud., vol. 105, Princeton Univ. Press, Princeton, NJ, 1983. MR0717034 (86b:49003)

[GM] M. Giaquinta and G. Modica, Remarks on the regularity of the minimizers of certain degenerate functionals, Manuscripta Math. 57 (1986), 55-99. MR0866406 (87m:49036) 
[Ma1] P. Marcellini, Regularity of minimizers of integrals of the calculus of variations with nonstandard growth conditions, Arch. Rational Mech. Anal. 10 (1989), 267-284. MR0969900 (90a:49017)

[Ma2] , Regularity for elliptic equations with general growth conditions, J. Differential Equations 105 (1993), 296-333. MR 1240398 (95h:35069)

[Ma3] , Everywhere regularity for a class of elliptic systems without growth conditions, Ann. Scuola Norm. Sup. Pisa Cl. Sci. (4) 23 (1996), 1-25. MR1401415 (97h:35048)

[Ma4] Regularity and existence of solutions of elliptic equations with ( $p, q)$-growth conditions, J. Differential Equations 90 (1991), 1-30. MR.1094446 (92e:35061)

[MP] P. Marcellini and G. Papi, Nonlinear elliptic systems with general growth, J. Differential Equations 221 (2006), 412-443. MR.2196484 (2007a:35024)

[MS] G. Mingione and F. Siepe, Full $C^{1, \alpha}$-regularity for minimizers of integral functionals with $L$ log L-growth, Z. Anal. Anwendungen 18 (1999), 1083-1100. MR.1736253 (2001f:49066)

[PS] A. Passarelli Di Napoli and F. Siepe, A regularity result for a class of anisotropic systems, Rend. Istit. Mat. Univ. Trieste 28 (1996), 13-31. MR.1463906 (98j:49052)

[Uh] K. Uhlenbeck, Regularity for a class of non-linear elliptic systems, Acta Math. 138 (1977), 219-240. MR0474389 (57:14031)

Universität des SaArlandes, Fachbereich 6.1 Mathematik, Postfach 1511 50, D-66041 SaArbrüCKen, Germany

E-mail address: fuchs@math.uni-sb.de

Received 8/AUG/2008

Originally published in English 